\title{
BMC medicine editorial board members on open access publishing
}

Michael R Carmont ${ }^{1}$, Stephen D Lawn², Babill Stray-Pedersen ${ }^{3}$, Yehuda Shoenfeld ${ }^{4}$ and Pascal Meier ${ }^{5}$

All correspondence should be made to the journal editorial office at bmcmedicineeditorial@biomedcentral.com

\begin{abstract}
In recognition of Open Access week (21st-27th October 2013), we asked some BMC Medicine Editorial Board Members to share their views and experiences on open access publishing. In this short video, they highlight the benefits of visibility and dissemination of their research, and discuss the future directions for this model of publishing.
\end{abstract}

\section{Additional file}

Additional file 1: BMC Medicine editorial board members talk on open access publishing.

\begin{abstract}
Author details
${ }^{1}$ Princess Royal Hospital, Shrewsbury and Telford NHS Trust and the Northern General Hospital, Sheffield Teaching Hospitals NHS Foundation Trust, Sheffield, UK. ${ }^{2}$ Department of Clinical Research, Faculty of Infectious and Tropical Diseases, London School of Hygiene and Tropical Medicine, Keppel Street, London, UK. ${ }^{3}$ Inst of Clinical Medicine, Oslo University and Div Women and children, Oslo University Hospital, Oslo, Norway. ${ }^{4}$ Zabludowicz Center for Autoimmune Diseases, Sheba Medical Center (Affiliated to Tel-Aviv University), Tel-Hashomer 52621, Israel. ${ }^{5}$ The Heart Hospital London, University College London Hospitals UCLH, London, UK.
\end{abstract}

Received: 14 October 2013 Accepted: 1 November 2013

Published: 21 Jan 2014

10.1186/1741-7015-12-10

Cite this article as: Carmont et al:: BMC medicine editorial board members on open access publishing. BMC Medicine 2014, 12:10

* Correspondence: bmcmedicineeditorial@biomedcentral.com

Full list of author information is available at the end of the article 